\title{
Are EWSR1-NFATc2-positive sarcomas really Ewing sarcomas?
}

\author{
Michaela C. Baldauf $^{1} \cdot$ Julia S. Gerke ${ }^{1} \cdot$ Martin F. Orth $^{1} \cdot$ Marlene Dallmayer $^{1} \cdot$ Daniel Baumhoer $^{2} \cdot$ Enrique de Alava $^{3}$. \\ Wolfgang Hartmann ${ }^{4} \cdot$ Thomas Kirchner ${ }^{5,6,7}$ - Thomas G. P. Grünewald $\mathbb{C D}^{1,5,6,7}$
}

Received: 13 November 2017 / Accepted: 19 December 2017

(c) United States \& Canadian Academy of Pathology 2018

Recently, Charville et al. [1] reported that EWSRI-rearranged fusion proteins mediate the expression of the pairedbox transcription factor $P A X 7$ in Ewing sarcoma. Based on an analysis of a published gene expression microarray dataset (accession code GSE60740), they state having identified $P A X 7$ to be significantly overexpressed in Ewing sarcoma in comparison to CIC-DUX4-positive round cell sarcomas [1]. In that microarray analysis they compared CIC-DUX4-positive sarcomas with EWSR1-NFATc2-positive sarcomas, assuming that EWSR1-NFATc2-positive sarcomas belong to the family of Ewing sarcomas [1], which are typically characterized by EWSRI-ETS fusion oncogenes [2]. Accordingly, Charville et al. [1] summarized in a schematic EWSR1-FLI1-, EWSR1-ERG-, and EWSR1$N F A T c 2$-positive sarcomas as "Ewing sarcoma," referring to Szuhai et al. (2009) [3], and did not take into account more recent reports in the literature that EWSR1-NFATc2-positive

Michaela C. Baldauf and Julia S. Gerke contributed equally to this work.

Thomas G. P. Grünewald

thomas.gruenewald@med.uni-muenchen.de

1 Max-Eder Research Group for Pediatric Sarcoma Biology, Institute of Pathology, Faculty of Medicine, LMU Munich, Munich, Germany

2 Bone Tumour Reference Center, Institute of Pathology, University Hospital Basel, University of Basel, Basel, Switzerland

3 Institute of Biomedicine of Seville (IBiS), Hospital Universitario Virgen del Rocío/CSIC/Universidad de Sevilla, CIBERONC, Seville, Spain

4 Gerhard-Domagk-Institute for Pathology, University Hospital Münster, Westfalian Wilhelms University, Münster, Germany

5 Institute of Pathology, Faculty of Medicine, LMU Munich, Munich, Germany

6 German Cancer Consortium (DKTK), Heidelberg, Germany

7 German Cancer Research Center (DKFZ), Heidelberg, Germany sarcomas may constitute an own entity [2]. Comparison of the dataset (GSE60740) used by Charville et al. to a published transcriptome reference dataset of genetically defined EWSRI-ETS-positive Ewing sarcomas (GSE34620) [4] and 13 other malignancies that may constitute morphological mimics [5] shows that EWSR1-NFATc2-positive sarcomas do not cluster with any other analyzed tumor entity including EWSR1-ETS-positive Ewing sarcoma (Fig. 1a).

Furthermore, Charville et al. noted that (in addition to PAX7) the genes FOXG1, NR5A2, SOX5, VDR, and TFAP2 were highly overexpressed in EWSR1-NFATC2-positive sarcomas as compared to CIC-DUX4-positive sarcomas [1]. Interestingly, all these genes are similarly lowly expressed in EWSR1-ETS-positive Ewing sarcomas and CIC-DUX4positive sarcomas (Fig. 1b). Collectively, these analyses strongly suggest that EWSR1-NFATC2-positive sarcomas constitute a tumor entity distinct from Ewing sarcoma [5]. Hence, the correctness of extrapolation from EWSR1NFATc2-positive sarcomas to Ewing sarcoma remains to be substantiated.

Based on their disputable extrapolation from EWSR1NFATc2-positive sarcomas to Ewing sarcomas, Charville et al. [1] performed immunohistochemical analyses of tissue samples, which were "diagnosed as Ewing sarcoma using a combination of morphologic, immunohistochemical, and molecular features." For the majority of cases (102/103), which were all "PAX7-positive" by immunohistochemistry, no details or references were provided on how the diagnosis of Ewing sarcoma was molecularly confirmed. Only for one sample, which was classified as "PAX7-negative," details were provided, but the diagnosis of Ewing sarcoma could not be molecularly confirmed [1]. The authors conclude from this sample set that "immunohistochemical detection of anti-PAX7 immunohistochemistry is a sensitive marker for Ewing sarcoma" [1]. Yet, it remains unclear whether the sensitivity of PAX7 is superior to that of the established Ewing sarcoma marker CD99 [6]. In their transcriptome analysis of EWSR1-NFATc2-positive sarcomas, Charville et al. noted that $C D 99$ was not significantly overexpressed 
a

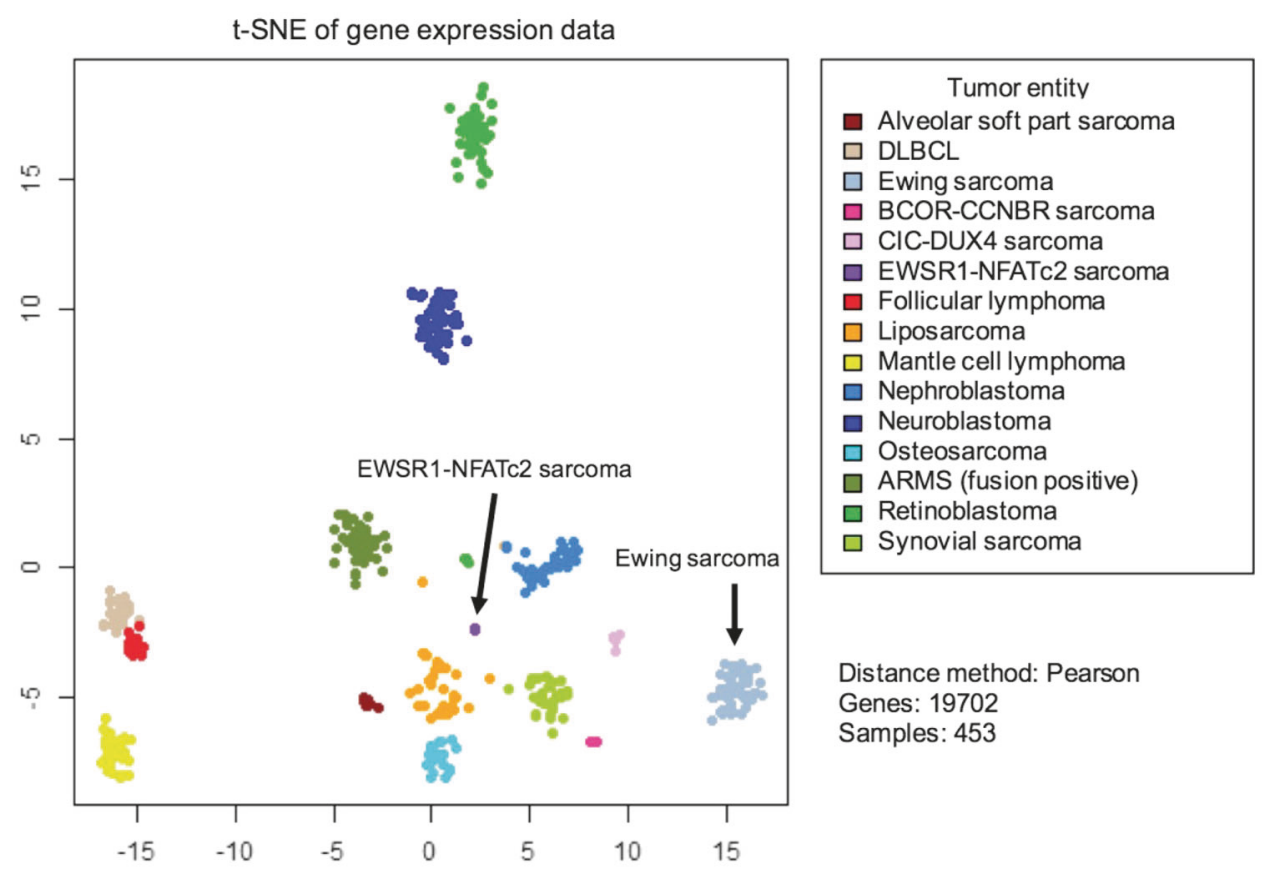

b
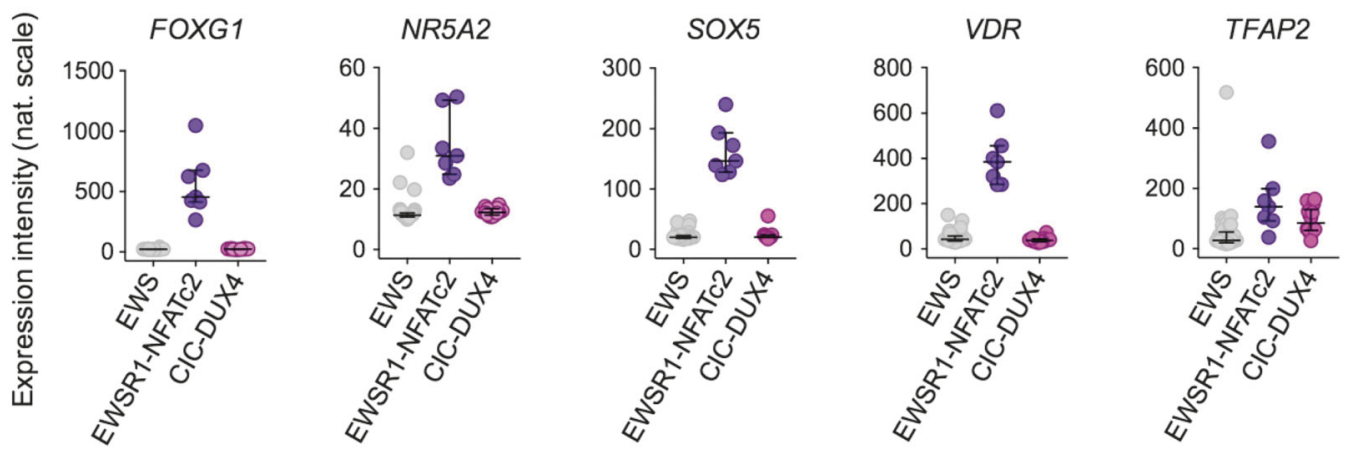

Fig. 1 EWSR1-NFATC2-positive sarcomas exhibit highly divergent transcriptomes from EWSR1-ETS-positive Ewing sarcomas. a t-SNE cluster analysis of Ewing sarcoma (EWSRl-ETS-positive) and other morphological mimics. All gene expression data were generated on Affymetrix HG-U133-Plus2.0 microarrays and simultaneously normalized using RMA and brainarray CDF [5], yielding one optimized probe-set per gene [13]. Gene expression data were filtered for a minimum tumor purity of $40 \%$ as determined by the ESTIMATE algorithm [14]. Principal component analysis (PCA) using all genes

compared to CIC-DUX4-positive sarcomas [1], supporting the concept that EWSR1-NFATc2-positive sarcomas are distinct from Ewing sarcoma, which typically shows high and uniform CD99 expression [6]. The proposed utility of PAX7 as a marker for Ewing sarcoma might be diminished given its limited specificity: In a previous report Charville et al. showed very high expression of PAX7 in morphological mimics such as embryonal, alveolar, and pleomorphic rhabdomyosarcomas, as well as synovial sarcomas [7], and in the current report they provide first evidence for its high expression in EWSR1-NFATC2-positive sarcomas [1]. represented on this microarray platform was done and visualized with the Rtsne Bioconductor package in $\mathrm{R}^{15}$. DLBCL diffuse large B-cell lymphoma, ARMS alveolar rhabdomyosarcoma. b Comparison of gene expression levels of FOXG1, NR5A2, SOX5, VDR, and TFAP2 in EWSR1-ETS-positive Ewing sarcomas (EWS), EWSR1-NFATc2-positive sarcomas, and CIC-DUX4-positive sarcomas (GSE34620 and GSE60740). Data are presented as dot plots. Horizontal bars indicate median expression levels. Whiskers indicate the interquartile range

Though PAX7 has been shown to be highly expressed in morphological mimics not positive for EWSRl-regarrangements such as rhabdomyosarcoma and synovial sarcoma [7], Charville et al. explain the high expression of $P A X 7$ in "Ewing sarcoma" by EWSR1-FLI1 binding to a GGAAmicrosatellite sequence located $20 \mathrm{~kb}$ telomeric to the PAX7 gene [1], which shows epigenetic characteristics of an active enhancer element in published ChIP-Seq data [8].

However, binding of EWSR1-FLI1 to this DNA sequence, EWSR1-FLI1-dependent enhancer activity, and a true interaction of this potential enhancer with the $P A X 7$ 
promoter were not confirmed experimentally. Yet, Charville et al. deduce from their approach that "these analyses provide evidence that a cis regulatory mechanism of EWSR1FLI1 binding and enhancer activation leads to expression of PAX7 in Ewing sarcoma" [1]. In the corresponding figure, two additional and more proximal EWSR1-FLI1-bound DNA elements are shown (the first mapping to another GGAA microsatellite, the second to a canonical ETS-like binding motif) with EWSR1-FLI1-dependent epigenetic characteristics of active enhancers, which were not further investigated or discussed by the authors [1]. Given that there are thousands of such EWSR1-FLI1-bound GGAA microsatellites in the vicinity of genes in Ewing sarcoma tumors $[8,9]$, it appears questionable to conclude that any EWSR1-FLI1-bound enhancer actually represents the cis regulatory element controlling the expression of a certain nearby gene. Previous reports showed that such EWSR1FLI1-bound GGAA microsatellites can loop to and accordingly regulate genes located in great distances (more than $300 \mathrm{~kb}$ ) [8]. Thus, the potential enhancer reported by Charville et al. could control the expression of any gene in cis or trans. Taking into account that GGAA microsatellites underlie substantial germline variability and that the enhancer activity of EWSR1-ETS-bound GGAA microsatellites strongly depends on the number of consecutive GGAA-repeats [10-12], it seems improbable that a highly polymorphic enhancer-like DNA element should lead to a uniformly high expression of $P A X 7$ in Ewing sarcoma as reported by Charville et al. [1]. Nevertheless, the authors conclude that EWSR1 fusion proteins are required for PAX7 expression in Ewing sarcoma by this mechanism. Given that ChIP-Seq analyses of Charville et al. are restricted to EWSR1-FLI1 [1], that there is as yet no convincing evidence that EWSR1-NFATc2 can bind to GGAA microsatellites as well, and that immunohistochemical analysis of PAX7 on EWSR1-NFATc2-positive sarcomas has not been performed, this conclusion is critical.

We deduce from the report of Charville et al. that particular care must be taken when analyzing published "omics"-data before conclusions may be drawn.

\section{Compliance with ethical standards}

Conflict of interest The authors declare that they have no conflict of interest.

\section{References}

1. Charville GW, Wang WL, Ingram DR, et al. EWSR1 fusion proteins mediate PAX7 expression in Ewing sarcoma. Mod Pathol. 2017;30:1312-20.

2. Kovar H, Amatruda J, Brunet E, et al. The second European interdisciplinary Ewing sarcoma research summit-a joint effort to deconstructing the multiple layers of a complex disease. Oncotarget. 2016;7:8613-24.

3. Szuhai K, Ijszenga M, de Jong D, et al. The NFATc2 gene is involved in a novel cloned translocation in a Ewing sarcoma variant that couples its function in immunology to oncology. Clin Cancer Res. 2009;15:2259-68.

4. Postel-Vinay S, Véron AS, Tirode F, et al. Common variants near TARDBP and EGR2 are associated with susceptibility to Ewing sarcoma. Nat Genet. 2012;44:323-7.

5. Baldauf MC, Orth MF, Dallmayer M, et al. Robust diagnosis of Ewing sarcoma by immunohistochemical detection of super-enhancer-driven EWSR1-ETS targets. Oncotarget. 2018;5:1587-601.

6. Shibuya R, Matsuyama A, Nakamoto M, et al. The combination of CD99 and NKX2.2, a transcriptional target of EWSR1-FLI1, is highly specific for the diagnosis of Ewing sarcoma. Virchows Arch. 2014;465:599-605.

7. Charville GW, Varma S, Forgó E, et al. PAX7 expression in rhabdomyosarcoma, related soft tissue tumors, and small round blue cell neoplasms. Am J Surg Pathol. 2016;40:1305-15.

8. Riggi N, Knoechel B, Gillespie SM, et al. EWS-FLI1 utilizes divergent chromatin remodeling mechanisms to directly activate or repress enhancer elements in Ewing sarcoma. Cancer Cell. 2014;26:668-81.

9. Tomazou EM, Sheffield NC, Schmidl C, et al. Epigenome mapping reveals distinct modes of gene regulation and widespread enhancer reprogramming by the oncogenic fusion protein EWSFLI1. Cell Rep. 2015;10:1082-95.

10. Grünewald TGP, Bernard V, Gilardi-Hebenstreit P, et al. Chimeric EWSR1-FLI1 regulates the Ewing sarcoma susceptibility gene EGR2 via a GGAA microsatellite. Nat Genet. 2015;47:1073-8.

11. Beck R, Monument MJ, Watkins WS, et al. EWS/FLI-responsive GGAA microsatellites exhibit polymorphic differences between European and African populations. Cancer Genet. 2012;205:304-12.

12. Monument MJ, Johnson KM, Mcllvaine E, et al. Clinical and biochemical function of polymorphic NR0B1 GGAAmicrosatellites in Ewing sarcoma: a report from the Children's Oncology Group. PLoS ONE. 2014;9:e104378.

13. Dai M, Wang P, Kostov G, et al. Evolving gene/transcript definitions significantly alter the interpretation of GeneChip data. Nucleic Acids Res. 2005;33:e175.

14. Yoshihara K, Shahmoradgoli M, Martinez E, et al. Inferring tumour purity and stromal and immune cell admixture from expression data. Nat Commun. 2013;4:2612.

15. van der Maaten L, Hinton G. Visualizing data using t-SNE. J Mach Learn Res. 2008;9:2579-605. 